Z Rheumatol $2021 \cdot 80: 755$

https://doi.org/10.1007/s00393-021-01094-0

Online publiziert: 28 . September 2021

(c) Springer Medizin Verlag $\mathrm{GmbH}$, ein Teil von

Springer Nature 2021

\section{Erratum zu: Sport und Bewegungstherapie bei entzündlichen rheumatischen Erkrankungen}

\author{
Wolfgang Hartung ${ }^{1} \cdot$ Philipp Sewerin ${ }^{2} \cdot$ Benedikt Ostendorf $^{3}$ \\ 'Asklepios Klinik Bad Abbach, Bad Abbach, Deutschland \\ ${ }^{2}$ Universitätsklinikum Düsseldorf, Düsseldorf, Deutschland \\ ${ }^{3}$ Rheumatologie am Hofgarten, Düsseldorf, Deutschland
}

\section{Erratum zu:}

Z Rheumatol 2021

https://doi.org/10.1007/s00393-021-

00970-z

Seite 255, Abschnitt: Sport und Bewegungstherapie bei Spondyloarthritiden:

In dem Abschnitt werden Studien aufgeführt, die für die axSpA (modifizierte New York-Kriterien) einen positiven Nutzen der Bewegungstherapie nachweisen.

Hier ist die Nomenklatur nicht exakt wiedergegeben. Bei den modifizierten New York-Kriterien handelt es sich richtigerweise um Klassifikationskriterien für die ankylosierende Spondylitis (AS) und nicht um Klassifikationskriterien oder Diagnosekriterien für die axSpA (axiale Spondyloarthritis). In die zitierten Studien wurden Patienten mit AS eingeschlossen.

Diese sachliche Ungenauigkeit bitten die Autoren zu entschuldigen.

Der Originalbeitrag wurde korrigiert.

Für die Autoren

Prof. W. Hartung

\section{Korrespondenzadresse}

Prof. Dr. med. Wolfgang Hartung

Asklepios Klinik Bad Abbach

Kaiser-Karl-V.-Allee 3, 93077 Bad Abbach,

Deutschland

w.hartung@asklepios.com
Die Online-Version des Originalartikels ist unter https://doi.org/10.1007/s00393-021-00970-z zu finden.

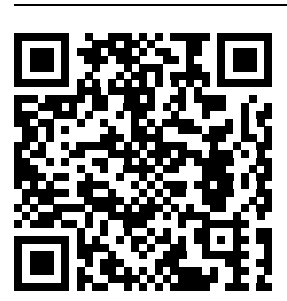

QR-Code scannen \& Beitrag online lesen 